\title{
Virtual Operations in Common Information Spaces: Boundary Objects and Practices
}

\author{
Demosthenes Akoumianakis, Giannis Milolidakis, Dimitrios Stefanakis, \\ Anargyros Akrivos, George Vellis, Dimitrios Kotsalis, Anargyros Plemenos, \\ and Nikolaos Vidakis \\ Department of Applied Information Technology \& Multimedia \\ TEI-Crete, Heraklion, Crete, Greece \\ Tel.: +30 2810379190 \\ $\{$ da , epp382, epp966, epp2063, epp646, epp665, epp1128 , \\ vidakis\} @epp. teicrete.gr
}

\begin{abstract}
The paper presents a field study aimed at identifying and analyzing the role of boundary artifacts in cross-organization virtual communities of practice $(\mathrm{CoP})$. Our analysis is informed by a recent case study in vacation package assembly (VPA), which is defined as the distributed collective practice carried out by members of a boundary-spanning virtual alliance inhabiting a 'common' information space (CIS). The CIS forms the virtuality through which members of the alliance engage in coordinative actions on boundary artifacts. The CIS implements the facilities required for constructing, negotiating and reconstructing these boundary artifacts so as to assemble personalized regional vacation packages for tourists. The results lead to several conclusions on the design of CIS as computational host of virtual communities of practice.
\end{abstract}

Keywords: Boundary objects, common information systems, virtual CoP.

\section{Introduction}

Building up collective experiences for improved knowledge-based work is important for geographically distributed organizations whose members are bound by a long or short-term common interest or goal, and who communicate and coordinate their work through information technology. In this vein, the present work aims to shed light to the design of tools for boundary-spanning virtual workgroups. In effect, we seek to advance an understanding of how cross-organization virtual alliances can be formed and facilitated by Common Information Spaces (CIS) (Bannon \& Bødker, 1997). In this context, a particular set of challenges seems to relate to the dual role members of these virtual alliances are engaged in (Lave \& Wenger, 1991) and the corresponding cognitive demands placed upon the CIS. Such duality arises from the fact that members of boundary-spanning virtual alliances are required, on the one hand to obey to their instituted 'local' practices and tools specific to their own business domain, and on the other hand to internalize and perform the 'shared' and 'collaborative' practice established and followed by the virtual alliance. As these constituent practices are frequently intertwined (i.e., local arrangements influence the members' collaborative 
behavior and praxis and vice versa), the cognitive demands placed upon the partners are high, while the design of the information infrastructure is typically complex and cumbersome.

This paper examines some of these challenges using a case study which describes distributed collective practices of boundary-spanning virtual alliances engaged in the assembly of vacation packages - a particular type of information-based product. Our interest is on the design of CIS so as to allow vacation packages to transcend across boundaries set either by competence-based electronic neighborhoods of the virtual alliance (i.e., accommodation, transportation, entertainment, food and beverage) or external and dynamic conditions, largely defined by online behavior and purchasing patterns of prospective customers.

The rest of the paper is structured as follows. The next section presents the theoretical base of the present work and elaborates on two concepts, namely CIS and boundary objects. Then, we present a case study on vacation package assembly in a cross-organization virtual alliance setting. We identify boundary artifacts and present details of their interactive manifestation in a CIS. The paper is concluded with a summary of key contributions and a discussion of their implications upon designing boundary practices in CIS.

\section{Theoretical Links}

The term 'common' or 'shared' information spaces (CIS) was introduced in (Bannon \& Bødker, 1997) to characterize information infrastructures which empower cooperating actors, engaged in interdependent activities of work, to coordinate their tasks so as to accomplish a collective objective. Subsequent refinements of the concept, mainly by CSCW scholars, have attempted either to establish links with communities of practice (Bossen, 2002) or to qualify parameters of CIS in terms of notions such as boundary objects (Star \& Griesemer, 1989), intermediary objects (Boujut \& Blanco, 2003), etc. Nevertheless, in the vast majority of cases CIS and their associated practices are examined in the context of single organizations. The more challenging problem of CIS crossing organizational boundaries - either through inter-organizational partnerships or external communities of practice - is seldom or loosely addressed (Dewhurst \& Cegarra Navarro, 2004). As our current intention is to consider cross-organizational collaboration in boundary spanning domains, it is of paramount importance to devise a suitable frame of reference to understand and facilitate such collaboration. To this effect, the boundary object concept offers a useful construct serving as 'language' or protocol for engagement in shared practices.

Since the introduction of the boundary object concept (Star \& Griesemer, 1989), researchers have explored the boundary role of a variety of artifacts such as diagrams, drawings, and blueprints (Bechky, 2003); workplace timelines and schedules such as Gantt and PERT charts in project-based work (Yakura, 2002); and digital documents (Murphy, 2001, Wegner, 1998; Brown \& Duguid, 1996; Bossen, 2002). Moreover, several studies have analyzed the role of boundary objects in domains such as translation (Bowker \& Star, 1999), micro-negotiations in CAD (Henderson, 1999), new product development (Carlile, 2002) as well as software development and HCI (Lutters \& Seaman, 2004). Despite the wide recognition, the boundary object concept is 
not without its critiques. Recently, CSCW scholars have questioned prevailing conceptions of boundary objects and have emphasized the need for a broader connotation for boundary artifacts (Carlile, 2006; Lutters \& Ackerman, 2007), extending the range of candidates to include information technologies such as document-centered groupware, organizational memory systems, social software and community management tools such as Blogs and Wikis.

Our current work shares common ground with these efforts as it aims to explore boundary artifacts as first class objects in CIS. By this account our intention is to shift the focus from the information processing characterizations of boundary artifacts (e.g., as repositories, diagrams, maps, etc) to the interaction affordances such artifacts should exhibit if they are to serve boundary practices in virtual space.

\section{Case Study}

The above research challenges are explored using a case study in building information-based vacation packages tightly coupled to a regional setting and offering added value to prospective visitors independently of pre-packaged holiday plan, choice of tour operator or destination management system. There are at least two possible perspectives on the added business value of these regional vacation packages - the first is derived from the packages' neutral role which makes it conceivable either as peripheral supplement to pre-packaged vacations or as a factor stimulating the ultimate choice of destination and/or pre-packaged solution; the second amounts to customers' increased capacity to exercise control and plan vacations in advance.

To gain insight to constructing vacation packages an exploratory survey was conducted utilizing interviews, on-site visits and scenarios to establish a context for design. As our intention was to unfold hidden or implicit elements of collaborative practices, interviews and on-site visits were tailored so as to feed envisioning of new (improved) practices. In turn, these were materialized using scenarios and rapid prototyping. Our survey was directed to tour operators, travel agencies and was complemented by documented codes of practice i.e., "Tour Operators Initiative". The findings led to insights on prevailing practices and the consolidation of an envisioned (virtual) practice.

\subsection{Current Practices}

Building vacation packages is instituted as a set of basic activities, such as defining package details and services, finding appropriate service providers, negotiating service details, finalizing agreements with service suppliers, promoting the package and package retailing. Some of these activities are administered individually (by one actor alone), while others entail a degree of cooperation and agreement between the involved parties. Moreover, depending on the nature of the vacation package, these activities may vary in scope and effort. For instance, package promotion subsumes different tasks for overseas vacation requiring special travel documents than domestic vacation where inland traveling is involved. As for the objects/artifacts of practice, a broad range was identified, including notebooks, drawing boards, schedules, etc, and a variety of IT tools facilitating communication (telephone, fax, e-mail, etc.), community support (i.e., portals and bulletin boards) as well as more advanced data-intensive 
operations (i.e., databases, repositories, reservation systems (CRS), customer relationship management (CRM) systems).

Our empirical evidence, as documented in the interviews, reveal that the above constrain both the types of vacation packages produced as well as the end users' testified willingness to exercise influence and plan vacations in advance. Moreover, although vacation package developers seem to recognize such constrains, they appear to be reluctant to introduce radical changes in the way the practice is conducted. These observations motivated the assessment of an envisioned practice designed to examine how a cross-organization virtual community of practice could cope with assembling rather than crafting vacation packages.

\subsection{Envisioning Practices in Virtual Settings}

Synthesizing the new practice entailed decisions on two primary constituents, namely the package development workflows (i.e., the practice domain) and community management. In terms of package development workflows, four distinct stages were identified, namely initiation, elaboration, deployment and tailoring, each hosting separate activities. Initiation amounts to the definition of the package, its duration and designation of the required services. Package elaboration entails negotiation and commitment of resources on behalf of the partners for the activities each can support. The deployment stage gathers all contributions and compiles them into a concrete offering which can be disseminated to prospective customers. Finally, the tailoring stage is concentrated on the package retailing which is geared to allowing customers to request further changes and modifications so as to suit own requirements.

The second constituent relates to the management of the cross-organization virtual alliance. For our purposes, the community of practice is conceived of as a missionspecific electronic squad. The mission is the development of the vacation package so as to meet designated constraints. The electronic squad is the cross-organization community of practice engaged in the vacation package development workflows. Three distinct roles are identified, namely the squad moderator, the squad member and the customer. This should be contrasted to the typical workgroup established in the traditional practice environment comprising the tour operator, the travel agent, the service providers and the end-customer. Moreover, as our squads are dynamic they follow a designated lifecycle. Our survey built upon existing sociological accounts rooted in dynamic group stabilization theories (Tuckman, 1965) to confirm four basic lifecycle stages, namely forming, storming, norming and performing.

The squad is formed once the package is defined in abstract terms. Squad reformation continues through the initiation workflow and up to the end of the elaboration workflow to allow candidates either to commit or opt out from a squad. At this point, the squad is stabilized and not likely to change until the end of the package creation lifecycle. The storming stage starts when initial activities are defined and the squad has taken its principal form. Storming is about reaching consensus on the specific mission and subsuming activities. The stage ends once all relevant issues have been addressed. Devising a common agenda with respect to the issues raised is the 
objective of the norming stage which is in partial overlap with the package elaboration workflow lasting until the end of the deployment workflow. Finally, the performing stage is in full temporal overlap with the package tailoring workflow.

\section{Common Information Space and Sense-Making}

This section describes components of the CIS which was designed to facilitate vacation package assembly as the distributed collective practice of an electronic squad. The emphasis is on the boundary artifacts used to make sense of the shared practice in virtual space and how such artifacts transcend different social worlds.

\subsection{Boundary Artifacts}

Vacation packages have two distinct properties - there are collective offerings with a prominent boundary function. Their 'collective' nature is derived from the fact that no single member of the virtual alliance can provide the package effectively and efficiently by account of own resources. On the other hand, their 'boundary' function is evident from the fact that vacation packages, as composite offerings as well as their constituent parts, should be recognizable by at least the following social groups: (a) different members of the cross-organization virtual alliance, each offering their own services such as accommodation, food \& beverage, transportation, etc., and (b) end user communities with different interests and preferences in the optional service offerings of the vacation package. Thus, they should be designed so as to transcend institutional boundaries resulting from the cross-organizational nature of the alliance and the exogenous boundaries implicitly set by different target end user communities. Recalling the definition of a boundary object by Star and Griesemer (1989, p. 393), some of the necessary qualities of these objects are that they should (a) be relevant and meaningful to different social worlds (b) have different meanings in different social worlds and c) have a common enough structure to make them recognizable in different social worlds. In our work, a social world is a neighborhood whose services are demanded by a vacation package. Thus, there are two types of boundary objects satisfying the above requirements of plasticity, namely neighborhood offerings (or activities) considered as primitive boundary objects and package families constituting composite boundary objects.
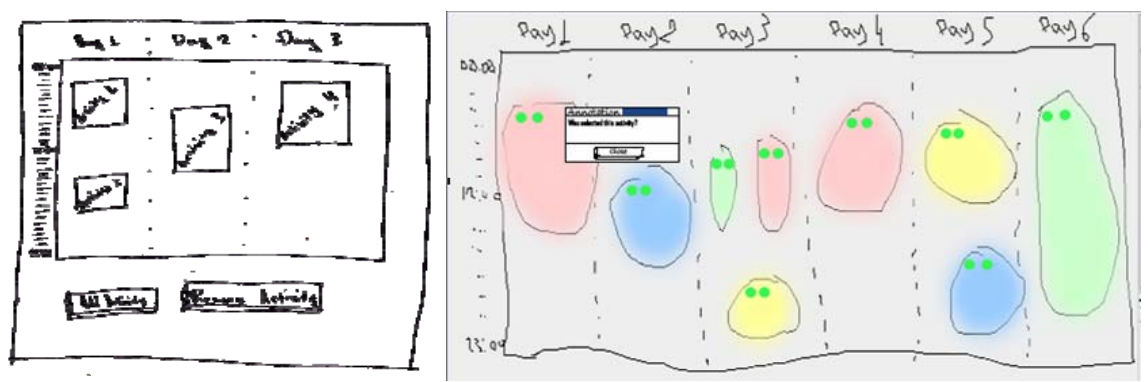

Fig. 1. Sketches of boundary objects with context information 
In an attempt to derive suitable symbolic representations of these boundary objects and their tractable properties, which would lead to an appropriate interactive manifestation in virtual settings, we used sketches and mock-ups to assess meaning and interpretive capacity across social worlds. Figure 1 presents examples of low-fidelity mockups of summative views of vacation packages and their primitive constituent activities. Rectangles represent neighborhood offerings which have specified type and duration in the context of a vacation package (i.e., panel). Color coding and social awareness indicators may be used to enhance the interpretive flexibility across collaborative contexts. Subsequent efforts aimed to further detail interaction-specific properties of these boundary objects as they transcend computing platforms, contexts of use and institutional boundaries.

\subsection{Spanning Internal Virtual Organization Boundaries}

Since neighborhood offerings are the primary constituents of a package, they need to be associated with a suitable interaction object class whose physical properties afford the intended meaning and interpretation. Following design deliberations, it was deemed appropriate to interactively manifest activity objects as customized 'elastic' buttons (with a label, color and size manipulation), inheriting the two-state dialogue model of a conventional button. The visual appearance of 'elastic' buttons is different from the typical, two-state GUI button. Specifically, their color designates neighborhood type, while direct manipulation of size allows indication of duration of the corresponding activity. To facilitate their interpretive flexibility, elastic buttons can host labels and icons to designate some of their features or current state. Furthermore, when rendered and manipulated in synchronous collaborative sessions, their dialog is enhanced to convey interim feedback through color and size variation as well as nested buttons for accepting / rejecting the current state values. These features were implemented by augmenting the Java Swing (Akoumianakis 2009).

Elastic buttons obtain their designated meaning when bundled into a suitable container which binds them to a vacation package. Flexible activity panels serve this purpose by utilizing dedicated layout management functions to organize neighborhood offerings (i.e., elastic buttons) in a particular layout. We have constructed two alternative layouts to represent role-specific (i.e., the moderator's and squad members') views of a vacation package. As shown in Figure 2, the moderator's view (lefthand side instance) concentrates on neighborhoods, while the squad members' view emphasizes activity timelines within days. It is worth noticing the representation of activities spanning across several days. In the moderator's view, these are represented as a uniform elastic button, whereas in the squad members view there are populated as a series of elastic buttons with slight modification of appearance to convey continuity of the offering across several days.

Some silent features of the implementation of the two activity panels include (a) the fact that at all times the panels present instances of the same object, although through different layout managers, (b) moderators have access to and can manipulate all components of an activity panel, while squad member belonging to a neighborhood can only manipulate objects of that neighborhood and (c) each activity operates in its own locale allowing automatic tailoring to the context of use and computing devise. 

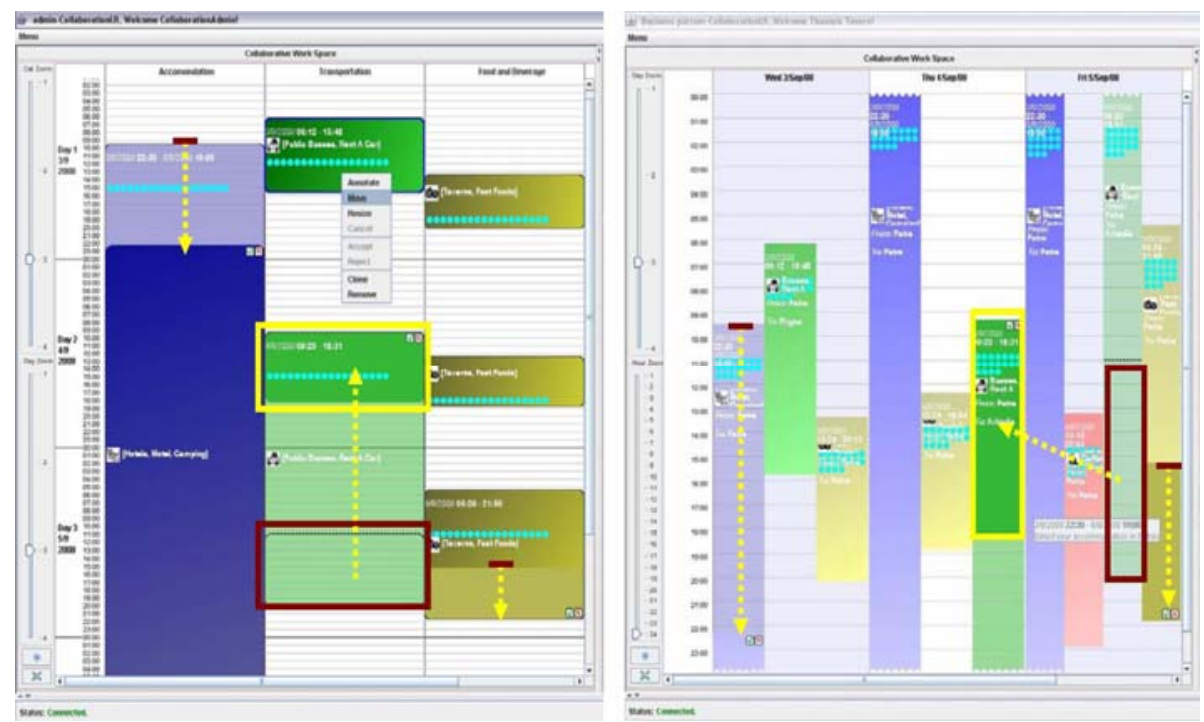

Fig. 2. Collaboration patterns in synchronous sessions

As vacation packages constitute 'collective' offerings their details are negotiated in the course of synchronous collaborative sessions. To this end, there are different versions of mutual awareness relevant, but due to space limitations we will briefly review the case of mutual awareness in synchronous collaboration sessions. A synchronous session is initiated by a moderator and allows members to negotiate aspects of a vacation package. The object of these negotiations is either part of or the entire package at a given point in time. However, as already mentioned, depending on their role different collaborators may view the same package differently and with different access rights. This requires object synchronization to ensure consistency of coordinative actions.

In the current implementation synchronization is achieved through managing distributed object replicas irrespective of their interactive manifestation. In Figure 2 the examples assume that the moderator (left screen) is the holder of the floor, thus the initiator of the collaborative tasks. The right screen depicts the effects of the initiator's actions on the collaborating partner's user interface The components marked in red and yellow represent start and end conditions respectively. The 'accept/reject' buttons are automatically introduced in the elastic buttons to indicate direction in the change of state. This allows collaborators, not only to make sense of current and proposed states, but also to express opinion and influence the ultimate details of the object of negotiation.

\subsection{Spanning External Boundaries: The Customers' Social Worlds}

Thus far we have examined how vacation packages span internal boundaries of the virtual workgroup as they are being constructed, negotiated and reconstructed. Nevertheless, the same objects once assembled are required to cross the boundary between 

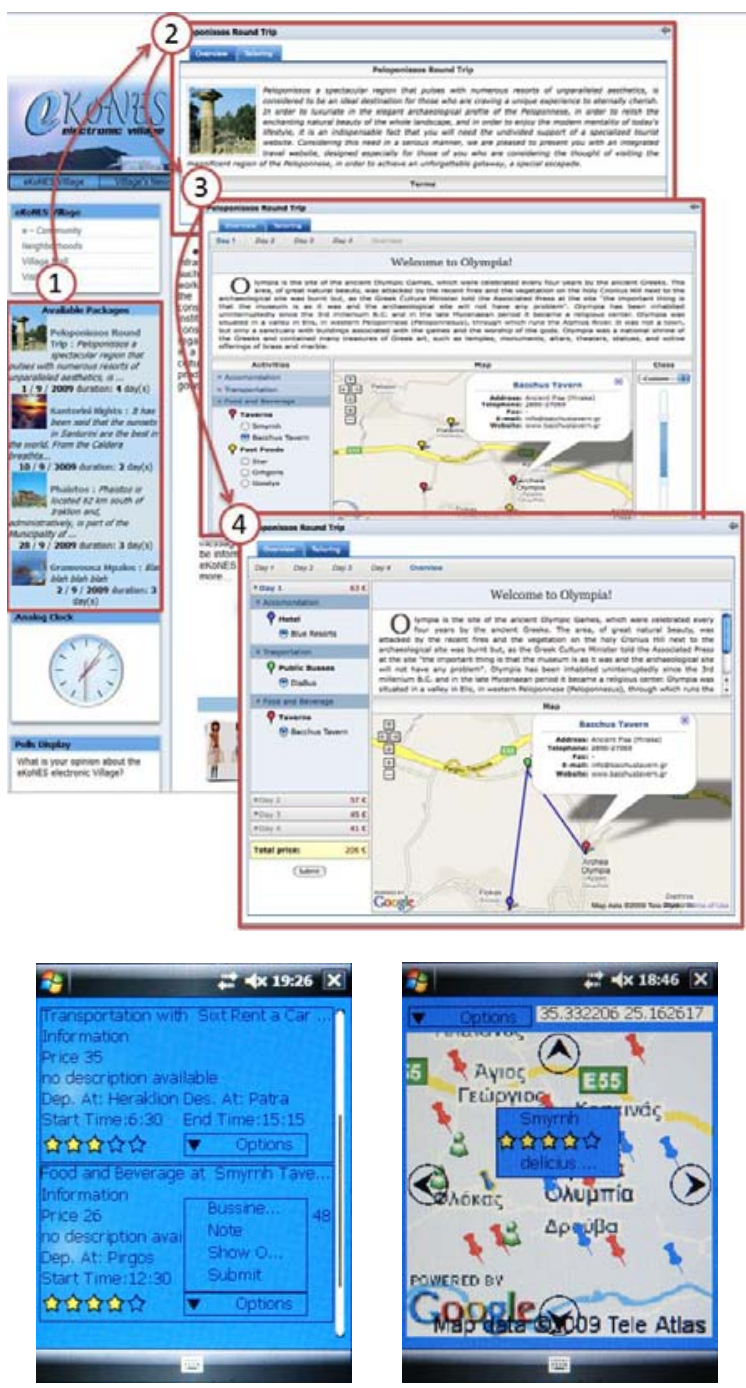

Fig. 3. Vacation packages in the customers' social world

the virtual workgroup and the customer base. To facilitate such crossing the assembly line undertakes to translate vacation packages in a language meaningful to the end users. The top image in Figure 3 depicts one type of such a translation illustrating how prospective users are informed of the package's availability (step 1) and prompted to consider making a personalized reservation (steps 2-4). The result of these interactions is fed back to the virtual workgroup, as request for tailoring, thus generating new cycles of meta-negotiations until a customized version of the package is derived. In a similar vein, while in vacation, customers may further reflect upon their experience with the package. The bottom images of Figure 3 depict how this is done using a mobile device. In the example the user has the ability to get an overall 
view of the services' details and their providers, as well as to add comments and rate the service quality. Such information is also fed back to the virtual work group facilitating meta-negotiations and perhaps improvements in subsequent versions of the package. Another useful provision in the mobile device context is derived by integrating the Google Maps services and the GPS of the device to allow users to find out the relative location of the supplier of a service on the map (i.e., the blue pin-like marker) and to become aware of the location of other customers (i.e., green pawn marks) that have registered in the same package family, thus strengthening ties between consumers of similar products.

\section{Consolidation and Contributions}

Guided by the need to understand the processes and mechanics of cooperation in cross-organization virtual alliances within the context of a virtual community of practice, the present work provides useful insights to fulfilling theoretical and engineering challenges. The theoretical challenge is concerned with understanding virtual practices and their constituent elements in online collaborative settings. On the other hand, the engineering challenge amounts to designing virtualities for social construction of boundary spanning knowledge and managing 'collective' artifacts. In terms of the theoretical challenge, the present work reveals that in virtual settings involving knowledge-based collaborative work, social interaction alone is not sufficient to reveal the constituents of practice in which collaborators engage in. In fact, a more thorough insight of the process, tools and the artifacts of work is required to make sense of what collaborators actually do and how the 'boundary' practice of the community intertwines with the collaborator's 'local' activities. The implication on engineering CIS is that they need to be designed so as to establish the 'place' for engaging in the practice the community is about. This extends current thinking on virtual CoP, which assumes that practice is revealed by analyzing the content of interactions in bulletin boards, threaded discussion forums and other types of community-support systems. Our experience indicates that, whatever practice may be unfolded through such systems, it will offer only partial insight to 'collaborative' praxis. A more informative account of what collaborators become engaged in requires re-framing practice beyond social interaction and into the activities subsumed by the boundary artifacts (i.e., the processes, objects and the tools) of cooperative work.

\section{Summary and Conclusions}

We have described the rationale of boundary artifacts as first class objects in a CIS devised to support vacation package assembly. Additionally, we have elaborated how these artifacts can be designed to exhibit plasticity and interpretive flexibility so as to facilitate sense making in virtual boundary-spanning settings. Collectively, the boundary objects, the tools for manipulating them and the shared workflow constitute the CIS for the designated practice of vacation package assembly. Recently completed and on-going virtual ethnographies of operating squads indicate a number of implications related both to the necessary quality attributes and the role of boundary objects in CIS (Akoumianakis, in print). Specifically, whereas for traditional boundary objects (e.g., drawings, forms and documents, repositories), interpretive flexibility, 
abstractness and plasticity suffice as necessary qualities, in the context of CIS boundary objects should additionally satisfy role-adaptability, replication, multiple view capability and view synchronization. As for the role of boundary objects in CIS, our work supports a notion of boundary artifacts as computer-mediated social practice vocabularies rather than mere translation devices.

\section{References}

Akoumianakis, D.: Managing Universal Accessibility Requirements in Software-intensive Projects. Software Process - Improvement \& Practice 14, 3-29 (2009)

Akoumianakis, D.: Electronic community factories: The model and its application in the tourism sector. Accepted for publication in Electronic Commerce Research. Springer, Heidelberg (in print)

Bannon, L., Bødker, S.: Constructing Common Information Spaces. In: Hughes, J., Rodden, T., Prinz, W., Schmidt, K. (eds.) ECSCW 1997: Proceedings of the 5th European CSCW Conference, pp. 91-96. Kluwer Academic Publishers, Dordrecht (1997)

Bossen, C.: The Parameters of Common Information Spaces: the Heterogeneity of Cooperative Work at a Hospital Ward. In: CSCW 2002, New Orleans, Louisiana, USA, November 16-20 (2002)

Boujut, J.-F., Blanco, E.: Intermediary Objects as a Means to Foster Co-operation in Engineering Design. Computer Supported Cooperative Work 12, 205-219 (2003)

Bowker, G.C., Star, S.L.S.: Sorting things out: classification and its consequences. MIT Press, Cambridge (1999)

Brown, J.S., Duguid, P.: The social life of documents. First Monday 1(1) (1996), http://www. firstmonday.dk/issues/issue1/documents /

Carlile, P.R.: A Pragmatic View of Knowledge and Boundaries: Boundary Objects in New Product Development. Organization Science 13, 442-455 (2002)

Carlile, P.R.: Artifacts and Knowledge Negotiation Across Domains. In: Rafaeli, A., Pratt, M.G. (eds.) Artifacts and Organizations: Beyond Mere Symbolism. LEA, Mahwah (2006)

Dewhurst, F.W., Cegarra Navarro, J.G.: External communities of practice and relational capital. The Learning Organization 11(4/5), 322-331 (2004)

Henderson, K.: On Line and On Paper: Visual Representations, Visual Culture, and Computer Graphics in Design Engineering. MIT Press, Cambridge (1999)

Lave, J., Wenger, E.: Situated Learning: Legitimate Peripheral Participation. Cambridge University Press, Cambridge (1991)

Lutters, W., Ackerman, M.: ) Beyond Boundary Objects: Collaborative Reuse in Aircraft Technical Support. Computer Supported Cooperative Work 16, 341-372 (2007)

Lutters, W.G., Seaman, C.B.: Software Maintenance and Support: Identifying Routine Work Artifacts as Boundary Objects Across Time (2004), IFIP 7.2, http: / /www.se-hci.org/bridging/chi2004/papers.html

Murphy, D.L.: Digital Documents in Organizational Communities of Practice: A First Look. In: 34th Hawaii International Conference on System Sciences. IEEE Computer Society, Los Alamitos (2001)

Star, S.L., Griesemer, J.: Institutional ecology, 'translations' and boundary objects. Social Studies of Science 19, 387-420 (1989)

Wegner, E.: Communities of Practice: Learning, Meaning and Identity. Cambridge University Press, Cambridge (1998)

Yakura, E.K.: Charting time: timelines as temporal boundary objects. Academy of Management Journal 45(5), 956-970 (2002) 